\title{
Mid-Truncated Burr XII distribution and its applications in order statistics
}

\author{
Jagdish Saran*, Narinder Pushkarna, Shikha Sehgal \\ Department of Statistics, University of Delhi, India
}

\begin{abstract}
In this paper we define the Mid-Truncated Burr XII distribution and derive some of its statistical properties such as moments, moment generating function, characteristic function, likelihood function, etc. We have also obtained the recurrence relations for single and product moments of order statistics in a random sample of size $\mathrm{n}$ drawn from MidTruncated Burr XII distribution. Further, the characterization of Mid-Truncated distributions using conditional moments has been given.
\end{abstract}

Keywords Order Statistics, Mid-Truncated Burr XII Distribution, Single Moments, Product Moments, Recurrence Relations, Incomplete Beta Function, Characterization

\section{AMS 2010 subject classifications 62G30}

DOI: $10.19139 /$ soic.v7i1.368

\section{Introduction}

Many authors like Malik [10], Balakrishnan and Joshi [1], Balakrishnan et al. [2], Saran and Pushkarna [17, 18, 19], etc. have obtained several results for the single and product moments of order statistics from the un-truncated, left truncated, right truncated and doubly truncated distributions. Mohie El-Din and Sultan [12] have obtained recurrence relations for moments of order statistics from doubly truncated continuous distributions. They have also presented some applications of their results. Mohie El-Din et al. [11] have obtained moment generating functions of order statistics from doubly truncated exponential distribution in terms of hypergeometric function. They have also derived some recurrence relations between these moment generating functions. The truncated distributions are quite effectively used where a random variable is restricted to be observed on some range and these situations are common in various fields. For instance, in survival analysis, failures during the warranty period may not be counted. Therefore many researchers were being attracted to the problem of analysing such truncated data encountered in various disciplines, proposed the truncated versions of the usual statistical distributions, to improve a forecasting actuarial model and particularly for modelling data from insurance policies. But there exist life models which do not obey the complete or truncated distribution, for example, in Microbiology (cf. Tortora et al. [21]), when a bacterial strain is inoculated into a liquid growth medium, the population is counted at intervals, it is possible to plot a bacterial growth curve. There are three basic phases of growth: the log, stationary, and death phases. During log phase, the bacterial cells are most metabolically active and are preferred for industrial purposes. During stationary phase we find that the number of cells will still remain constant due to the number of bacterial death balances with the number of new cells. Consequently during this phase no investigation is required. After the stationary phase the most bacterial cells will die because of the exclusion of nutrients and lead to accumulation of waste products. In such live models mid-truncated distributions are quite effectively used where the random variable is restricted

\footnotetext{
${ }^{*}$ Correspondence to: Jagdish Saran (Email: jagdish_saran52@yahoo.co.in). Department of Statistics, University of Delhi, Delhi 110007,
} India.

ISSN 2310-5070 (online) ISSN 2311-004X (print)

Copyright (C) 2019 International Academic Press 
to be observed on some sub intervals of the given specified range (cf. Okasha et al. [14] and Mohie El-Din et al. [13]).

Burr system of distributions was constructed in 1942 by Irving W. Burr [3]. Since the corresponding density functions have a wide variety of shapes, this system is useful for approximating histograms, particularly when a simple mathematical structure for the fitted cumulative distribution function (cdf) is required. Other applications include simulation, quantal response, approximation of distributions, and development of non-normal control charts. Pathak and Chaturvedi [15] evaluated reliability function for Burr (X) distribution for its wide variety of application in life time data. Recently work on optimal sample size determination for Burr (XII) population has been done by Hashempour [5]. According to Soliman [20], this distribution covers the curve shape characteristic for a large number of distributions. Due to versatility and flexibility of the Burr (XII) distribution, this distribution is quite attractive as a tentative model for data whose underlying distribution is unknown.

In this paper, we propose to consider the mid-truncated Burr distribution (MTBD) (Type XII) with three parameters, which contains, as special sub models, mid-truncated Weibull distribution, mid-truncated exponential distribution, mid-truncated log-logistic distribution, among others. Due to the flexibility of the considered distribution in accommodating different forms of risk function, this MTBD is appropriate for a variety of problems modelling lifetime data.

\section{Mid-truncated distribution}

We define the Mid-truncated distribution as follows:

Let $Y$ be a continuous random variable with baseline probability density function (pdf) $g(y)$, cumulative distribution function (cdf) $G(y)$, define $X$ as a corresponding mid-truncated variable, of the random variable $Y$ with pdf $f(x)$. We define

$$
f(x)=\left\{\begin{array}{ll}
p \frac{g(x)}{G\left(Q_{1}\right)}, & \text { if } x \leq Q_{1} \\
q \frac{g(x)}{1-G\left(P_{1}\right)}, & \text { if } x \geq P_{1}
\end{array}, \quad 0 \leq p \leq 1,0 \leq q \leq 1, \text { s.t. } p+q=1, Q_{1} \leq P_{1},\right.
$$

which is called the Mid-truncated density function and, $Q_{1}$ and $P_{1}$ are the points of mid truncation of the baseline distribution under consideration. Also we assume that

$$
Q=\int_{-\infty}^{Q_{1}} g(x) d x
$$

and

$$
1-P=\int_{P_{1}}^{\infty} g(x) d x
$$

Then equation (2.1) can be written as

$$
f(x)=\left\{\begin{array}{ll}
p \frac{g(x)}{Q}, & \text { if } x \leq Q_{1} \\
q \frac{g(x)}{1-P}, & \text { if } x \geq P_{1}
\end{array}, 0 \leq p \leq 1,0 \leq q \leq 1, \text { s.t. } p+q=1, Q_{1} \leq P_{1} .\right.
$$

\section{1. $k$-th moment}

The $k$-th moment of a mid-truncated random variable $X$ (denoted by $\mu_{*}^{(k)}$ ) is given by:

$$
\mu_{*}^{(k)}=\frac{p}{G\left(Q_{1}\right)} \int_{-\infty}^{Q_{1}} x^{k} g(x) d x+\frac{q}{1-G\left(P_{1}\right)} \int_{P_{1}}^{\infty} x^{k} g(x) d x .
$$


Integrating by parts, we get

$$
\mu_{*}^{(k)}=\frac{p}{G\left(Q_{1}\right)}\left[Q_{1}^{k} G\left(Q_{1}\right)-\int_{-\infty}^{Q_{1}} k x^{k-1} G(x) d x\right]+\frac{q}{\left(1-G\left(P_{1}\right)\right)}\left[\mu^{(k)}-P_{1}^{k} G\left(P_{1}\right)+\int_{-\infty}^{P_{1}} k x^{k-1} G(x) d x\right],
$$

where $\mu^{(k)}$ is the $k$-th moment of the un-truncated distribution.

\subsection{Moment generating function and characteristic function}

The moment generating function of a mid-truncated random variable $X$ (denoted by $M^{*}(t)$ ) is given by:

$$
M^{*}(t)=\frac{p}{G\left(Q_{1}\right)} \int_{-\infty}^{Q_{1}} e^{x t} g(x) d x+\frac{q}{1-G\left(P_{1}\right)} \int_{P_{1}}^{\infty} e^{t x} g(x) d x .
$$

Integrating by parts, we get

$$
M^{*}(t)=p e^{t Q_{1}}-\frac{p}{G\left(Q_{1}\right)} \int_{-\infty}^{Q_{1}} t e^{x t} G(x) d x+\frac{q}{1-G\left(P_{1}\right)}\left[M(t)-e^{t P_{1}} G\left(P_{1}\right)+\int_{-\infty}^{P_{1}} t e^{t x} G(x) d x\right],
$$

where $M(t)$ is the moment generating function of the un-truncated random variable.

Similarly, the characteristic function of the mid-truncated random variable is given by:

$$
\Phi^{*}(t)=p e^{i t Q_{1}}-\frac{p}{G\left(Q_{1}\right)} \int_{-\infty}^{Q_{1}} i t e^{i x t} G(x) d x+\frac{q}{1-G\left(P_{1}\right)}\left[\Phi(t)-e^{i t P_{1}} G\left(P_{1}\right)+\int_{-\infty}^{P_{1}} i t e^{i t x} G(x) d x\right],
$$

where $\Phi(t)$ is the characteristic function of the un-truncated random variable.

\subsection{Distribution function}

The distribution function of a mid-truncated random variable $X$ is given by:

$$
F(x)= \begin{cases}p \frac{G(x)}{G\left(Q_{1}\right)}, & -\infty<x \leq Q_{1} \\ 1-q \frac{1-G(x)}{1-G\left(P_{1}\right)}, & P_{1} \leq x<\infty .\end{cases}
$$

\subsection{Generating data}

If the distribution function of the mid-truncated random variable exists we can use the inverse transform method to generate the data. To generate a random variable $X$ with distribution function $F(x)$ as given in (2.7), draw $U_{1}, U_{2}$ from $U(0,1)$ and then solve each of the following equations

$$
u_{1}=\frac{G(x)}{G\left(Q_{1}\right)}, \quad u_{2}=\left(1-\frac{1-G(x)}{1-G\left(P_{1}\right)}\right)
$$

with respect to $x$.

\subsection{Likelihood function}

For $n$ observations $x_{1}, x_{2}, \ldots, x_{n}$ from a mid-truncated distribution, the likelihood function is given by:

$$
\begin{aligned}
& L(\theta)=\prod_{i=1}^{r} \frac{p g\left(x_{i}\right)}{G\left(Q_{1}\right)} \prod_{i=r+1}^{n} \frac{q g\left(x_{i}\right)}{\left(1-G\left(P_{1}\right)\right)}, \text { where } r \text { is the number of } x_{i} \text { 's } \leq Q_{1} . \\
& L(\theta)=\frac{p^{r} q^{n-r}}{\left(G\left(Q_{1}\right)\right)^{r}\left(1-G\left(P_{1}\right)\right)^{n-r}} \prod_{i=1}^{n} g\left(x_{i}\right) .
\end{aligned}
$$




\section{Mid-truncated Burr XII distribution}

The probability density function (pdf) of Burr distribution (type XII) is given by

$$
g(x)=\nu \beta \lambda x^{\beta-1}\left(1+\lambda x^{\beta}\right)^{-(\nu+1)}, \quad 0 \leq x<\infty, \nu, \beta, \lambda>0,
$$

and the cumulative distribution function (cdf) is given by

$$
G(x)=1-\left(1+\lambda x^{\beta}\right)^{-\nu}, \quad 0 \leq x<\infty, \nu, \beta, \lambda>0 .
$$

Then the probability density function (pdf) of mid-truncated Burr distribution (type XII) is given by

$$
f(x)= \begin{cases}\frac{p \nu \beta \lambda x^{\beta-1}\left(1+\lambda x^{\beta}\right)^{-(\nu+1)}}{Q}, & 0<x \leq Q_{1} \\ \frac{q \nu \beta \lambda x^{\beta-1}\left(1+\lambda x^{\beta}\right)^{-(\nu+1)}}{1-P}, & P_{1} \leq x<\infty\end{cases}
$$

and the cumulative distribution function (cdf) is given by

$$
F(x)= \begin{cases}\frac{p\left(1-\left(1+\lambda x^{\beta}\right)^{-\nu}\right)}{Q}, & 0<x \leq Q_{1} \\ 1-\frac{q\left(1+\lambda x^{\beta}\right)^{-\nu}}{1-P}, & P_{1} \leq x<\infty .\end{cases}
$$

Using (3.3) and (3.4), we get the relation between pdf and cdf as

$$
1-F(x)= \begin{cases}1-\frac{p}{Q}+f(x) \frac{x^{1-\beta}\left(1+\lambda x^{\beta}\right)}{\nu \beta \lambda}, & 0<x \leq Q_{1} \\ f(x) \frac{x^{1-\beta}\left(1+\lambda x^{\beta}\right)}{\nu \beta \lambda}, & P_{1} \leq x<\infty .\end{cases}
$$

Using (2.2) and (2.3), we get

$$
\begin{aligned}
& Q_{1}=\left\{\frac{(1-Q)^{\frac{-1}{\nu}}-1}{\lambda}\right\}^{\frac{1}{\beta}} \\
& P_{1}=\left\{\frac{(1-P)^{\frac{-1}{\nu}}-1}{\lambda}\right\}^{\frac{1}{\beta}} .
\end{aligned}
$$

Using (3.6) and (3.7), equations (3.3) and (3.4) can be rewritten as

$$
f(x)= \begin{cases}\frac{p \nu \beta \lambda x^{\beta-1}\left(1+\lambda x^{\beta}\right)^{-(\nu+1)}}{1-\left(1+\lambda Q_{1}^{\beta}\right)^{-\nu}}, & 0<x \leq Q_{1} \\ \frac{q \nu \beta \lambda x^{\beta-1}\left(1+\lambda x^{\beta}\right)^{-(\nu+1)}}{\left(1+\lambda P_{1}^{\beta}\right)^{-\nu}}, & P_{1} \leq x<\infty,\end{cases}
$$

and

$$
F(x)= \begin{cases}\frac{p\left(1-\left(1+\lambda x^{\beta}\right)^{-\nu}\right)}{1-\left(1+\lambda Q_{1}^{\beta}\right)^{-\nu}}, & 0<x \leq Q_{1} \\ 1-\frac{q\left(1+\lambda x^{\beta}\right)^{-\nu}}{\left(1+\lambda P_{1}^{\beta}\right)^{-\nu}}, & P_{1} \leq x<\infty .\end{cases}
$$


The mid-truncated Burr XII density function for $\beta=2, \lambda=4, \nu=0.5, p=q=0.5$ truncated at $Q_{1}=1.5$ and $P_{1}=3.25$ is provided in Figure 1.

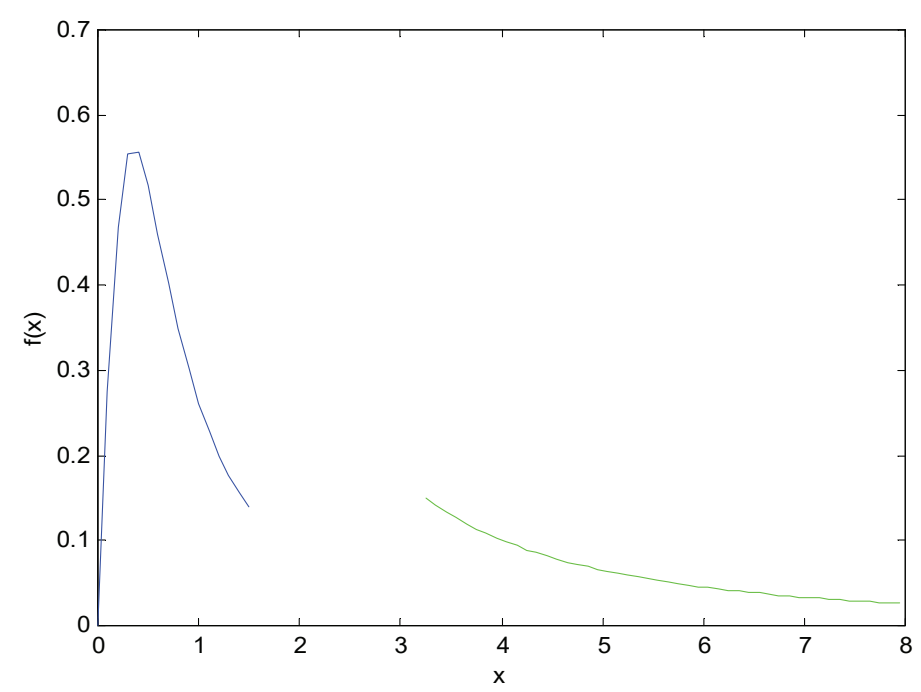

Figure 1. Probability density function for mid-truncated Burr XII distribution.

The mid-truncated Burr XII density function for $\beta=2.6, \lambda=0.5, \nu=2, p=0.8$ and $q=0.2$ truncated at $Q_{1}=1.9$ and $P_{1}=5.9$ is provided in Figure 2.

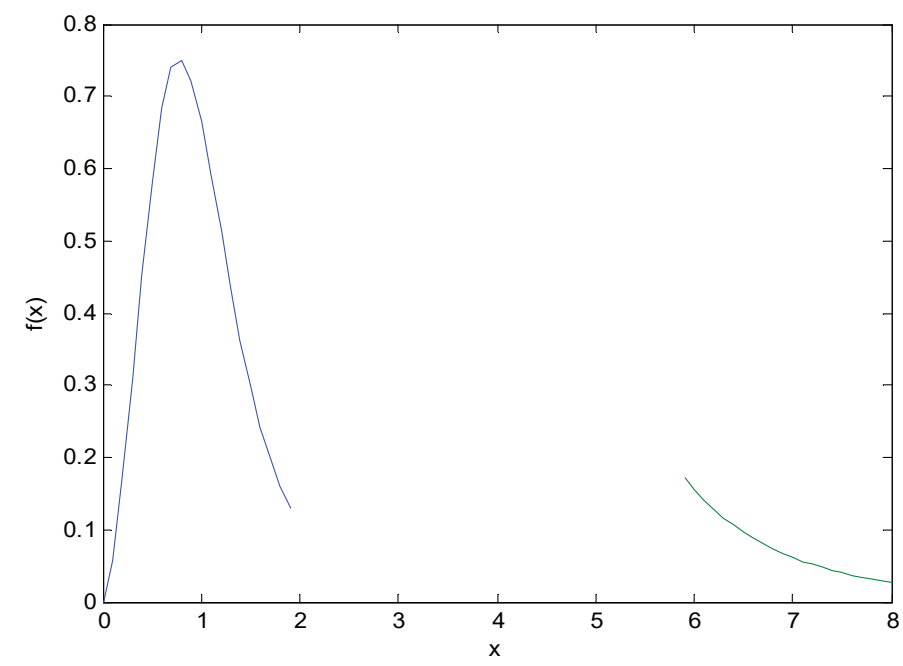

Figure 2. Probability density function for mid-truncated Burr XII distribution.

Let $X_{1}, X_{2}, \ldots, X_{n}$ be a random sample of size $n$ from the mid-truncated Burr XII distribution defined in (3.3) and let $X_{1: n} \leq X_{2: n} \leq \ldots \leq X_{n: n}$ be the corresponding order statistics. Thus the probability density function (pdf) of $X_{r: n}(1 \leq r \leq n)$ is given by:

$$
f_{r: n}(x)=c_{r: n}[F(x)]^{r-1}[1-F(x)]^{n-r} f(x), \quad 0 \leq x<\infty,
$$


where $c_{r: n}=\frac{n !}{(r-1) !(n-r) !}$.

The joint density function of order statistics $X_{r: n}$ and $X_{s: n}(1 \leq r<s \leq n)$ is given by

$$
f_{r, s: n}(x, y)=c_{r, s: n}[F(x)]^{r-1}[F(y)-F(x)]^{s-r-1}[1-F(y)]^{n-s} f(y) f(x), \quad 0 \leq x<y<\infty,
$$

where

$$
c_{r, s: n}=\frac{n !}{(r-1) !(s-r-1) !(n-s) !}
$$

(cf. David and Nagaraja [4]).

The single moments of order statistics $X_{r: n}(1 \leq r \leq n)$ are given by

$$
\mu_{r: n}^{(k)}=E\left(X_{r: n}^{k}\right)=\int_{0}^{Q_{1}} x^{k} f_{r: n}(x) d x+\int_{P_{1}}^{\infty} x^{k} f_{r: n}(x) d x, k=0,1,2, \ldots
$$

Similarly, the product moments of $X_{r: n}$ and $X_{s: n}(1 \leq r<s \leq n)$ are given by

$$
\mu_{r, s: n}^{(j, k)}=E\left(X_{r: n}^{j} X_{s: n}^{k}\right)=\int_{0}^{Q_{1}} \int_{x}^{Q_{1}} x^{j} y^{k} f_{r, s: n}(x, y) d y d x+\int_{P_{1}}^{\infty} \int_{x}^{\infty} x^{j} y^{k} f_{r, s: n}(x, y) d y d x .
$$

\section{1. $k$-th moment}

The $k$-th moment of the mid-truncated Burr XII distribution can be obtained using (2.5) and is given by:

$$
\mu_{*}^{(k)}=\frac{p}{G\left(Q_{1}\right)}\left[Q_{1}^{k} G\left(Q_{1}\right)-k \int_{0}^{Q_{1}} x^{k-1} G(x) d x\right]+\frac{q}{\left(1-G\left(P_{1}\right)\right)}\left[\mu^{(k)}-P_{1}^{k} G\left(P_{1}\right)+k \int_{0}^{P_{1}} x^{k-1} G(x) d x\right] .
$$

Substituting the values of $G\left(Q_{1}\right), G\left(P_{1}\right)$ and $G(x)$ from equation (3.2) and solving, we get

$$
\begin{aligned}
\mu_{*}^{(k)}= & \frac{p}{G\left(Q_{1}\right)}\left[-Q_{1}^{k}\left(1+\lambda Q_{1}^{\beta}\right)^{-\nu}\right]+\frac{q}{\left(1-G\left(P_{1}\right)\right)}\left[\mu^{(k)}+P_{1}^{k}\left(1+\lambda P_{1}^{\beta}\right)^{-\nu}\right] \\
& +\frac{p k}{G\left(Q_{1}\right)} \int_{0}^{Q_{1}} x^{k-1}\left(1+\lambda x^{\beta}\right)^{-\nu} d x-\frac{q k}{1-G\left(P_{1}\right)} \int_{0}^{P_{1}} x^{k-1}\left(1+\lambda x^{\beta}\right)^{-\nu} d x \\
= & \frac{p}{G\left(Q_{1}\right)}\left[-Q_{1}^{k}\left(1+\lambda Q_{1}^{\beta}\right)^{-\nu}\right]+\frac{q}{\left(1-G\left(P_{1}\right)\right)}\left[\mu^{(k)}+P_{1}^{k}\left(1+\lambda P_{1}^{\beta}\right)^{-\nu}\right] \\
& +\frac{p k}{G\left(Q_{1}\right)} \frac{\beta_{m\left(Q_{1}\right)}\left(\frac{k}{\beta}, \nu-\frac{k}{\beta}\right)}{\beta \lambda^{\beta}}-\frac{q k}{1-G\left(P_{1}\right)} \frac{\beta_{m\left(P_{1}\right)}\left(\frac{k}{\beta}, \nu-\frac{k}{\beta}\right)}{\beta \lambda^{\beta}},
\end{aligned}
$$

where $m\left(Q_{1}\right)=\frac{\lambda Q_{1}{ }^{\beta}}{1+\lambda Q_{1}{ }^{\beta}}, m\left(P_{1}\right)=\frac{\lambda P_{1}{ }^{\beta}}{1+\lambda P_{1}{ }^{\beta}}$ and $\beta_{x}(a, b)=\int_{0}^{x} t^{a-1}(1-t)^{b-1} d t, 0<x<1$, is the incomplete beta function and can be obtained using tables given by Pearson et al. [16].

To investigate the effect of the shape parameters $\beta, \nu$ and scale parameter $\lambda$ on the mid-truncated Burr XII density function we have computed mean, variance, skewness and kurtosis for different values of the parameters, which are presented in following Tables 1 to 3.

Table $1 . \nu=6$ and $\lambda=1.5$

\begin{tabular}{|l|c|c|c|c|}
\hline \hline$\beta$ & \multicolumn{1}{|c|}{ Mean } & Variance & Skewness $\left(\beta_{1}\right)$ & Kurtosis $\left(\beta_{2}\right)$ \\
\hline 0.8 & 2.2156 & 5.5589 & 1.1701 & 10.6781 \\
\hline 0.9 & 2.1373 & 4.7813 & 0.487 & 4.7715 \\
\hline 0.95 & 2.1076 & 4.5052 & 0.3348 & 3.6722 \\
\hline 1 & 2.0826 & 4.2775 & 0.2375 & 2.9912 \\
\hline 1.5 & 1.9648 & 3.1544 & 0.0195 & 1.3656 \\
\hline 2 & 1.939 & 2.7109 & 0.0033 & 1.1638 \\
\hline \hline
\end{tabular}


Table 2. $\nu=5$ and $\beta=1$

\begin{tabular}{|l|c|c|c|c|}
\hline \hline \multicolumn{1}{|c|}{$\lambda$} & Mean & Variance & Skewness $\left(\beta_{1}\right)$ & Kurtosis $\left(\beta_{2}\right)$ \\
\hline 1 & 2.2716 & 5.1371 & 0.9897 & 8.3788 \\
\hline 1.23 & 2.2294 & 5.0299 & 0.8434 & 7.5447 \\
\hline 2 & 2.1553 & 4.8732 & 0.6276 & 6.2366 \\
\hline 2.5 & 2.1309 & 4.829 & 0.5663 & 5.846 \\
\hline 3 & 2.1144 & 4.8009 & 0.5274 & 5.5938 \\
\hline 4 & 2.0937 & 4.7674 & 0.481 & 5.2883 \\
\hline \hline
\end{tabular}

Table 3. $\beta=1$ and $\lambda=1.5$

\begin{tabular}{|l|c|c|c|c|}
\hline \hline$\nu$ & Mean & Variance & Skewness $\left(\beta_{1}\right)$ & Kurtosis $\left(\beta_{2}\right)$ \\
\hline 5 & 2.1953 & 4.9532 & 0.7379 & 6.9181 \\
\hline 6 & 2.0826 & 4.2775 & 0.2375 & 2.9912 \\
\hline 6.5 & 2.0412 & 4.0658 & 0.15 & 2.3661 \\
\hline 7 & 2.0067 & 3.9016 & 0.0996 & 1.9957 \\
\hline 8 & 1.9523 & 3.6639 & 0.049 & 1.5964 \\
\hline 10 & 1.8796 & 3.3818 & 0.0159 & 1.283 \\
\hline \hline
\end{tabular}

\subsection{Moment generating function and characteristic function}

The moment generating function of the mid-truncated Burr XII distribution is given by:

$$
M^{*}(t)=\frac{p}{G\left(Q_{1}\right)} \int_{0}^{Q_{1}} e^{x t} g(x) d x+\frac{q}{1-G\left(P_{1}\right)} \int_{P_{1}}^{\infty} e^{t x} g(x) d x .
$$

Integrating by parts, we get

$$
\begin{aligned}
M^{*}(t) & =p e^{t Q_{1}}-\frac{p}{G\left(Q_{1}\right)} \int_{0}^{Q_{1}} t e^{x t} G(x) d x+\frac{q}{1-G\left(P_{1}\right)}\left[M(t)-e^{t P_{1}} G\left(P_{1}\right)+\int_{0}^{P_{1}} t e^{t x} G(x) d x\right] \\
& =p e^{t Q_{1}}+\frac{q}{1-G\left(P_{1}\right)}\left[M(t)-e^{t P_{1}} G\left(P_{1}\right)\right]-\frac{p t}{G\left(Q_{1}\right)} \int_{0}^{Q_{1}} e^{x t} G(x) d x+\frac{q t}{1-G\left(P_{1}\right)} \int_{0}^{P_{1}} e^{t x} G(x) d x .
\end{aligned}
$$

Substituting the value of $G(x)=1-\left(1+\lambda x^{\beta}\right)^{-\nu}$ from (3.2), and solving we get

$$
\begin{aligned}
M^{*}(t)= & p e^{t Q_{1}}+\frac{q}{1-G\left(P_{1}\right)}\left[M(t)-e^{t P_{1}} G\left(P_{1}\right)\right]-\frac{p}{G\left(Q_{1}\right)}\left(e^{t Q_{1}}-1\right)+\frac{q}{1-G\left(P_{1}\right)}\left(e^{t P_{1}}-1\right) \\
& +\frac{p t}{G\left(Q_{1}\right)} \sum_{m=0}^{\infty} \frac{t^{m}}{m !} \int_{0}^{Q_{1}} x^{m}\left(1+\lambda x^{\beta}\right)^{-\nu} d x-\frac{q t}{1-G\left(P_{1}\right)} \sum_{m=0}^{\infty} \frac{t^{m}}{m !} \int_{0}^{P_{1}} x^{m}\left(1+\lambda x^{\beta}\right)^{-\nu} d x \\
= & p e^{t Q_{1}}+\frac{q}{1-G\left(P_{1}\right)}\left[M(t)-e^{t P_{1}} G\left(P_{1}\right)\right]-\frac{p}{G\left(Q_{1}\right)}\left(e^{t Q_{1}}-1\right)+\frac{q}{1-G\left(P_{1}\right)}\left(e^{t P_{1}}-1\right) \\
& +\frac{p t}{G\left(Q_{1}\right)} \sum_{d=0}^{\infty} \frac{t^{d}}{d !} \frac{\beta_{m\left(Q_{1}\right)}\left(\frac{d+1}{\beta}, \nu-\frac{d+1}{\beta}\right)}{\beta \lambda^{(d+1) / \beta}}-\frac{q t}{1-G\left(P_{1}\right)} \sum_{d=0}^{\infty} \frac{t^{d}}{d !} \frac{\beta_{m\left(P_{1}\right)}\left(\frac{d+1}{\beta}, \nu-\frac{d+1}{\beta}\right)}{\beta \lambda^{(d+1) / \beta}},
\end{aligned}
$$

where $m\left(Q_{1}\right)=\frac{\lambda Q_{1}{ }^{\beta}}{1+\lambda Q_{1}{ }^{\beta}}, m\left(P_{1}\right)=\frac{\lambda P_{1}{ }^{\beta}}{1+\lambda P_{1}{ }^{\beta}}$ and $\beta_{x}(a, b)=\int_{0}^{x} t^{a-1}(1-t)^{b-1} d t, 0<x<1$, is the incomplete beta function and can be obtained using tables given by Pearson et al. [16].

Similarly the characteristic function of the mid-truncated Burr XII distribution can be evaluated. 


\subsection{Distribution function}

The cumulative distribution function of the mid-truncated Burr XII distribution (as in equation (3.9)) is given by

$$
F(x)=\left\{\begin{array}{cl}
p \frac{1-\left(1+\lambda x^{\beta}\right)^{-\nu}}{1-\left(1+\lambda Q_{1}^{\beta}\right)^{-\nu}}, & 0<x \leq Q_{1} \\
1-q \frac{\left(1+\lambda x^{\beta}\right)^{-\nu}}{\left(1+\lambda P_{1}^{\beta}\right)^{-\nu}}, & P_{1} \leq x<\infty .
\end{array}\right.
$$

\subsection{Generating data}

To generate random variables from mid-truncated Burr XII distribution, we use equations (3.14) and (2.8), and we get

$$
\begin{aligned}
& u_{1}=\frac{1-\left(1+\lambda x^{\beta}\right)^{-\nu}}{1-\left(1+\lambda Q_{1}^{\beta}\right)^{-\nu}}, \\
& u_{2}=1-\frac{\left(1+\lambda x^{\beta}\right)^{-\nu}}{\left(1+\lambda P_{1}^{\beta}\right)^{-\nu}} .
\end{aligned}
$$

Solving the above two equations for $x$, we get

$$
x= \begin{cases}{\left[\frac{\left[1-u_{1}\left(1-\left(1+\lambda Q_{1}^{\beta}\right)^{-\nu}\right)\right]^{-1 / \nu}-1}{\lambda}\right]^{1 / \beta},} & 0<x \leq Q_{1} \\ {\left[\frac{\left[\left(1-u_{2}\right)\left(1+\lambda P_{1}^{\beta}\right)^{-\nu}\right]^{-1 / \nu}-1}{\lambda}\right]^{1 / \beta},} & P_{1} \leq x<\infty .\end{cases}
$$

\subsection{Likelihood function}

For $n$ observations $x_{1}, x_{2}, \ldots, x_{n}$ from mid-truncated Burr XII distribution the likelihood function is obtained from equation (2.9) and is given by:

$$
\begin{aligned}
L(\nu, \beta, \lambda)= & \frac{p^{r} q^{n-r}}{\left(1-\left(1+\lambda Q_{1}{ }^{\beta}\right)^{-\nu}\right)^{r}\left(\left(1+\lambda P_{1}{ }^{\beta}\right)^{-\nu}\right)^{(n-r)}} \prod_{i=1}^{n} \nu \beta \lambda x_{i}{ }^{\beta-1}\left(1+\lambda x_{i}{ }^{\beta}\right)^{-(\nu+1)} \\
\log L(\nu, \beta, \lambda)= & r \log (p)+(n-r) \log (q)+n(\log \nu+\log \beta+\log \lambda)+(\beta-1) \sum_{i=1}^{n} \log \left(x_{i}\right) \\
& \quad-(\nu+1) \sum_{i=1}^{n} \log \left(1+\lambda x_{i}^{\beta}\right)-r \log \left(1-\left(1+\lambda Q_{1}^{\beta}\right)^{-\nu}\right)+\nu(n-r) \log \left(1+\lambda P_{1}^{\beta}\right) .
\end{aligned}
$$

\section{Recurrence relations for single moments of order statistics from mid-truncated Burr XII distribution}

In this section we will derive recurrence relations for single moments of order statistics from mid-truncated Burr XII distribution as defined in Section 3.

Theorem 1

For $k=\beta, \nu>1$ and $\lambda, \beta>0$, we have

$$
\mu_{1: n}^{(\beta)}\left(1-\frac{1}{n \nu}\right)=\frac{(Q-p) \beta}{Q} I_{1: n}^{(\beta)}-q^{n}\left[\left\{\frac{(1-Q)^{\frac{-1}{\nu}}-1}{\lambda}\right\}-\left\{\frac{(1-P)^{\frac{-1}{\nu}}-1}{\lambda}\right\}\right]
$$


and, for $k>\beta, \beta \in Z^{+}, \nu>1$ and $\lambda, \beta>0$, we have

$$
\mu_{1: n}^{(k)}=\frac{k}{n \nu \beta \lambda} \mu_{1: n}^{(k-\beta)}+\frac{k}{n \nu \beta} \mu_{1: n}^{(k)}+\frac{(Q-p) k}{Q} I_{1: n}^{(k)}-q^{n}\left[\left[\left\{\frac{(1-Q)^{\frac{-1}{\nu}}-1}{\lambda}\right\}^{\frac{1}{\beta}}\right]^{k}-\left[\left\{\frac{(1-P)^{\frac{-1}{\nu}}-1}{\lambda}\right\}^{\frac{1}{\beta}}\right]^{k}\right],
$$

where

$$
I_{1: n}^{(k)}= \begin{cases}\frac{Q_{1}^{k}}{k}, & n=1 \\
\left\{1+\sum_{j=1}^{n-1}(-1)^{j}\left(\frac{p}{Q}\right)^{j}\left(\begin{array}{c}
n-1 \\
j
\end{array}\right)\right\} \frac{Q_{1}^{k}}{k}+\sum_{j=1}^{n-1} \sum_{i=1}^{j}(-1)^{j+i}\left(\frac{p}{Q}\right)^{j}\left(\begin{array}{c}
n-1 \\
j
\end{array}\right)\left(\begin{array}{c}
j \\
i
\end{array}\right) \frac{\beta_{m\left(Q_{1}\right)}\left(\frac{k}{\beta}, \nu i-\frac{k}{\beta}\right)}{\beta \lambda^{k / \beta}}, & n>1,\end{cases}
$$

$I_{1: n}^{(\beta)}$ is the same as $I_{1: n}^{(k)}$ with $k$ replaced by $\beta, m\left(Q_{1}\right)=\frac{\lambda Q_{1}{ }^{\beta}}{1+\lambda Q_{1}{ }^{\beta}}$ and $\beta_{x}(a, b)=\int_{0}^{x} t^{a-1}(1-t)^{b-1} d t, 0<x<1$, is the incomplete beta function of first kind and can be obtained by using tables given by Pearson et al. [16].

Proof

Relations in (4.1) and (4.2) may be proved by following exactly the same steps as those in proving Theorem 2 which is presented below.

\section{Theorem 2}

For $2 \leq r \leq n, k=\beta, \nu>1, \lambda, \beta>0$, we have

$$
\mu_{r: n}^{(\beta)}\left(1-\frac{1}{n \nu}\right)-\mu_{r-1: n-1}^{(\beta)}=\frac{(Q-p) \beta}{Q} I_{r: n}^{(\beta)}-\left(\begin{array}{c}
n-1 \\
r-1
\end{array}\right) p^{r-1} q^{n-r+1}\left[\left\{\frac{(1-Q)^{\frac{-1}{\nu}}-1}{\lambda}\right\}-\left\{\frac{(1-P)^{\frac{-1}{\nu}}-1}{\lambda}\right\}\right],
$$

and, for $k>\beta, \beta \in Z^{+}, \nu>1, \lambda, \beta>0$, we have

$$
\begin{aligned}
\mu_{r: n}^{(k)}-\mu_{r-1: n-1}^{(k)}= & \frac{k}{n \nu \beta \lambda} \mu_{r: n}^{(k-\beta)}+\frac{k}{n \nu \beta} \mu_{r: n}^{(k)}+\frac{(Q-p) k}{Q} I_{r: n}^{(k)} \\
& -\left(\begin{array}{c}
n-1 \\
r-1
\end{array}\right) p^{r-1} q^{n-r+1}\left[\left[\left\{\frac{(1-Q)^{\frac{-1}{\nu}}-1}{\lambda}\right\}^{\frac{1}{\beta}}\right]^{k}-\left[\left\{\frac{(1-P)^{\frac{-1}{\nu}}-1}{\lambda}\right\}^{\frac{1}{\beta}}\right]^{k}\right]
\end{aligned}
$$

where

$$
I_{r: n}^{(k)}= \begin{cases}\sum_{j=r}^{n} \sum_{i=1}^{j-1}(-1)^{j-r+i}\left(\frac{p}{Q}\right)^{j-1}\left(\begin{array}{c}
n-1 \\
j-1
\end{array}\right)\left(\begin{array}{c}
j-1 \\
r-1
\end{array}\right)\left(\begin{array}{c}
j-1 \\
i
\end{array}\right) \frac{\beta_{m\left(Q_{1}\right)}\left(\frac{k}{\beta}, \nu i-\frac{k}{\beta}\right)}{\beta \lambda^{k / \beta}}, & 2 \leq r<n \\
\left(\frac{p}{Q}\right)^{n-1}\left\{\frac{Q_{1}^{k}}{k}+\sum_{i=1}^{n-1}(-1)^{i}\left(\begin{array}{c}
n-1 \\
i
\end{array}\right) \frac{\beta_{m\left(Q_{1}\right)}\left(\frac{k}{\beta}, \nu i-\frac{k}{\beta}\right)}{\beta \lambda^{k / \beta}}\right\}, & r=n,\end{cases}
$$

$I_{r: n}^{(\beta)}$ is the same as $I_{r: n}^{(k)}$ with $k$ replaced by $\beta, m\left(Q_{1}\right)=\frac{\lambda Q_{1}{ }^{\beta}}{1+\lambda Q_{1}{ }^{\beta}}$ and

$$
\beta_{x}(a, b)=\int_{0}^{x} t^{a-1}(1-t)^{b-1} d t, \quad 0<x<1,
$$

is the incomplete beta function of first kind and can be obtained by using tables by Pearson et al. [16].

Proof

To prove the above theorem, first we prove the following two lemmas. 


\section{Lemma 1}

In usual notations, for an arbitrary mid-truncated distribution discussed in Section 2, we have

$$
\begin{aligned}
\mu_{r: n}^{(k)}-\mu_{r-1: n-1}^{(k)}=\left(\begin{array}{c}
n-1 \\
r-1
\end{array}\right) & {\left[k \int_{0}^{Q_{1}} x^{k-1}(F(x))^{r-1}(1-F(x))^{n-r+1} d x\right.} \\
& \left.+k \int_{P_{1}}^{\infty} x^{k-1}(F(x))^{r-1}(1-F(x))^{n-r+1} d x-\left(Q_{1}{ }^{k}-P_{1}{ }^{k}\right) p^{r-1} q^{n-r+1}\right] .
\end{aligned}
$$

Proof

Using (3.12), we get

$$
\begin{aligned}
\mu_{r: n}^{(k)}-\mu_{r-1: n-1}^{(k)}= & C_{r: n} \int_{0}^{Q_{1}} x^{k}(F(x))^{r-1}(1-F(x))^{n-r} f(x) d x \\
& +C_{r: n} \int_{P_{1}}^{\infty} x^{k}(F(x))^{r-1}(1-F(x))^{n-r} f(x) d x \\
& -C_{r-1: n-1} \int_{0}^{Q_{1}} x^{k}(F(x))^{r-2}(1-F(x))^{n-r} f(x) d x \\
& -C_{r-1: n-1} \int_{P_{1}}^{\infty} x^{k}(F(x))^{r-2}(1-F(x))^{n-r} f(x) d x \\
= & \left(\begin{array}{c}
n-1 \\
r-1
\end{array}\right)\left[\int_{0}^{Q_{1}} x^{k}(F(x))^{r-2}(1-F(x))^{n-r} f(x)\{n F(x)-(r-1)\} d x\right. \\
& \left.\quad+\int_{P_{1}}^{\infty} x^{k}(F(x))^{r-2}(1-F(x))^{n-r} f(x)\{n F(x)-(r-1)\} d x\right] .
\end{aligned}
$$

Let

$$
\varphi(x)=-(F(x))^{r-1}(1-F(x))^{n-r+1} .
$$

Differentiating both sides with respect to $x$, we get

$$
\frac{d \varphi(x)}{d x}=(F(x))^{r-2}(1-F(x))^{n-r} f(x)\{n F(x)-(r-1)\} .
$$

On using (4.8), equation (4.6) becomes

$$
\begin{aligned}
\mu_{r: n}^{(k)}-\mu_{r-1: n-1}^{(k)} & =\left(\begin{array}{c}
n-1 \\
r-1
\end{array}\right)\left[\int_{0}^{Q_{1}} x^{k}\left(\frac{d \varphi(x)}{d x}\right) d x+\int_{P_{1}}^{\infty} x^{k}\left(\frac{d \varphi(x)}{d x}\right) d x\right] \\
& =\left(\begin{array}{c}
n-1 \\
r-1
\end{array}\right)\left[\left[x^{k} \varphi(x)\right]_{0}^{Q_{1}}-k \int_{0}^{Q_{1}} x^{k-1} \varphi(x) d x+\left[x^{k} \varphi(x)\right]_{P_{1}}^{\infty}-k \int_{P_{1}}^{\infty} x^{k-1} \varphi(x) d x\right] \\
& =\left(\begin{array}{c}
n-1 \\
r-1
\end{array}\right)\left[Q_{1}^{k} \varphi\left(Q_{1}\right)-P_{1}^{k} \varphi\left(P_{1}\right)-k \int_{0}^{Q_{1}} x^{k-1} \varphi(x) d x-k \int_{P_{1}}^{\infty} x^{k-1} \varphi(x) d x\right] .
\end{aligned}
$$

Now, since $F\left(Q_{1}\right)=p$ and $F\left(P_{1}\right)=1-q$, therefore, from (4.7), we have

$$
\varphi\left(Q_{1}\right)=\varphi\left(P_{1}\right)=-p^{r-1} q^{n-r+1} .
$$

Substituting the values of $\varphi(x), \varphi\left(Q_{1}\right)$ and $\varphi\left(P_{1}\right)$ in (4.9), it leads to Lemma 1.

\section{Lemma 2}

If for $1 \leq r \leq n$ and $k=1,2, \ldots$,

$$
I_{r: n}^{(k)}=\left(\begin{array}{c}
n-1 \\
r-1
\end{array}\right) \int_{0}^{Q_{1}} x^{k-1}(F(x))^{r-1}(1-F(x))^{n-r} d x,
$$


then, it may be written as

$I_{1: n}^{(k)}= \begin{cases}\frac{Q_{1}^{k}}{k}, & n=1 \\ \left\{1+\sum_{j=1}^{n-1}(-1)^{j}\left(\frac{p}{Q}\right)^{j}\left(\begin{array}{c}n-1 \\ j\end{array}\right)\right\} \frac{Q_{1}^{k}}{k}+\sum_{j=1}^{n-1} \sum_{i=1}^{j}(-1)^{j+i}\left(\frac{p}{Q}\right)^{j}\left(\begin{array}{c}n-1 \\ j\end{array}\right)\left(\begin{array}{c}j \\ i\end{array}\right) \frac{\beta_{m\left(Q_{1}\right)}\left(\frac{k}{\beta}, \nu i-\frac{k}{\beta}\right)}{\beta \lambda^{k / \beta}}, & n>1,\end{cases}$

and

$$
I_{r: n}^{(k)}= \begin{cases}\sum_{j=r}^{n} \sum_{i=1}^{j-1}(-1)^{j-r+i}\left(\frac{p}{Q}\right)^{j-1}\left(\begin{array}{c}
n-1 \\
j-1
\end{array}\right)\left(\begin{array}{c}
j-1 \\
r-1
\end{array}\right)\left(\begin{array}{c}
j-1 \\
i
\end{array}\right) \frac{\beta_{m\left(Q_{1}\right)}\left(\frac{k}{\beta}, \nu i-\frac{k}{\beta}\right)}{\beta \lambda^{k / \beta}}, & 2 \leq r<n \\
\left(\frac{p}{Q}\right)^{n-1}\left\{\frac{Q_{1}^{k}}{k}+\sum_{i=1}^{n-1}(-1)^{i}\left(\begin{array}{c}
n-1 \\
i
\end{array}\right) \frac{\beta_{m\left(Q_{1}\right)}\left(\frac{k}{\beta}, \nu i-\frac{k}{\beta}\right)}{\beta \lambda^{k / \beta}}\right\}, & r=n,\end{cases}
$$

where $m\left(Q_{1}\right)=\frac{\lambda Q_{1}{ }^{\beta}}{1+\lambda Q_{1}{ }^{\beta}}$ and $\beta_{x}(a, b)=\int_{0}^{x} t^{a-1}(1-t)^{b-1} d t, 0<x<1$, is the incomplete beta function of first kind, which can be found out using tables given by Pearson et al. [16].

Proof

Relation in (4.11) may be proved by following exactly the same steps as those in proving (4.12) which is presented below.

Expanding $(1-F(x))^{n-r}$ binomially in powers of $F(x)$ and then substituting the same in (4.10), we get for $2 \leq r<n$,

$$
I_{r: n}^{(k)}=\left(\begin{array}{c}
n-1 \\
r-1
\end{array}\right) \sum_{l=0}^{n-r}(-1)^{l}\left(\begin{array}{c}
n-r \\
l
\end{array}\right) \int_{0}^{Q_{1}} x^{k-1}(F(x))^{l+r-1} d x .
$$

On putting $j=l+r$, we get

$$
I_{r: n}^{(k)}=\left(\begin{array}{c}
n-1 \\
r-1
\end{array}\right) \sum_{j=r}^{n}(-1)^{j-r}\left(\begin{array}{c}
n-r \\
j-r
\end{array}\right) \int_{0}^{Q_{1}} x^{k-1}(F(x))^{j-1} d x .
$$

Substituting the value of $F(x)$ from (3.4), we get

$$
\begin{aligned}
I_{r: n}^{(k)} & =\sum_{j=r}^{n}(-1)^{j-r}\left(\begin{array}{c}
n-1 \\
j-1
\end{array}\right)\left(\begin{array}{l}
j-1 \\
r-1
\end{array}\right) \int_{0}^{Q_{1}} x^{k-1}\left(\frac{p\left(1-\left(1+\lambda x^{\beta}\right)^{-\nu}\right)}{Q}\right)^{j-1} d x \\
& =\sum_{j=r}^{n}(-1)^{j-r}\left(\begin{array}{c}
n-1 \\
j-1
\end{array}\right)\left(\begin{array}{l}
j-1 \\
r-1
\end{array}\right) \frac{p^{j-1}}{Q^{j-1}} \int_{0}^{Q_{1}} x^{k-1}\left(\left(1-\left(1+\lambda x^{\beta}\right)^{-\nu}\right)\right)^{j-1} d x .
\end{aligned}
$$

Expanding $\left(\left(1-\left(1+\lambda x^{\beta}\right)^{-\nu}\right)\right)^{j-1}$ binomially, we get

$$
\begin{aligned}
I_{r: n}^{(k)} & =\sum_{j=r}^{n}(-1)^{j-r}\left(\begin{array}{c}
n-1 \\
j-1
\end{array}\right)\left(\begin{array}{c}
j-1 \\
r-1
\end{array}\right) \frac{p^{j-1}}{Q^{j-1}} \sum_{i=1}^{j-1}\left(\begin{array}{c}
j-1 \\
i
\end{array}\right) \int_{0}^{Q_{1}}(-1)^{i} x^{k-1}\left(1+\lambda x^{\beta}\right)^{-\nu i} d x \\
& =\sum_{j=r}^{n} \sum_{i=1}^{j-1}(-1)^{j-r+i}\left(\frac{p}{Q}\right)^{j-1}\left(\begin{array}{c}
n-1 \\
j-1
\end{array}\right)\left(\begin{array}{c}
j-1 \\
r-1
\end{array}\right)\left(\begin{array}{c}
j-1 \\
i
\end{array}\right) \int_{0}^{Q_{1}} x^{k-1}\left(1+\lambda x^{\beta}\right)^{-\nu i} d x .
\end{aligned}
$$


Now consider

$$
\begin{aligned}
J & =\int_{0}^{Q_{1}} x^{k-1}\left(1+\lambda x^{\beta}\right)^{-\nu i} d x \\
& =\frac{\int_{1}^{1+\lambda Q_{1}^{\beta}} y^{-\nu i}(y-1)^{\frac{k}{\beta}-1} d y}{\beta \lambda^{k / \beta}}, \text { by taking } y=1+\lambda x^{\beta} \\
& =\frac{1}{\beta \lambda^{k / \beta}} \int_{0}^{\frac{\lambda Q_{1} \beta}{1+\lambda Q_{1} \beta}} z^{\frac{k}{\beta}-1}(1-z)^{\nu i-\frac{k}{\beta}-1} d z, \text { by taking } z=(y-1) / y \\
& =\frac{1}{\beta \lambda^{k / \beta}} \beta_{m\left(Q_{1}\right)}\left(\frac{k}{\beta}, \nu i-\frac{k}{\beta}\right),
\end{aligned}
$$

where $m\left(Q_{1}\right)=\frac{\lambda Q_{1}{ }^{\beta}}{1+\lambda Q_{1}{ }^{\beta}}$ and $\beta_{x}(a, b)=\int_{0}^{x} t^{a-1}(1-t)^{b-1} d t, 0<x<1$, is the incomplete beta function and can be obtained using tables given by Pearson et al. [16].

Substituting the value of $J$ from (4.14) in (4.13), it leads to the result (4.12) for the case $2 \leq r<n$. The result (4.12) for the case $r=n$ can similarly be established.

\section{Proof of the main Theorem 2}

Using (3.5) in (4.5), we get

$$
\begin{aligned}
\mu_{r: n}^{(k)}-\mu_{r-1: n-1}^{(k)}= & \left(\begin{array}{c}
n-1 \\
r-1
\end{array}\right)\left[k \int_{0}^{Q_{1}} x^{k-1}(F(x))^{r-1}(1-F(x))^{n-r}\left(1-\frac{p}{Q}+f(x) \frac{x^{1-\beta}\left(1+\lambda x^{\beta}\right)}{\nu \beta \lambda}\right) d x\right. \\
& \left.+k \int_{P_{1}}^{\infty} x^{k-1}(F(x))^{r-1}(1-F(x))^{n-r} f(x) \frac{x^{1-\beta}\left(1+\lambda x^{\beta}\right)}{\nu \beta \lambda} d x-\left(Q_{1}^{k}-P_{1}^{k}\right) p^{r-1} q^{n-r+1}\right] \\
= & \left(\begin{array}{c}
n-1 \\
r-1
\end{array}\right)\left[k \frac{(Q-p)}{Q} \int_{0}^{Q_{1}} x^{k-1}(F(x))^{r-1}(1-F(x))^{n-r} d x\right. \\
& +\frac{k}{\nu \beta \lambda}\left\{\int_{0}^{Q_{1}} x^{k-\beta}(F(x))^{r-1}(1-F(x))^{n-r} f(x) d x\right. \\
& \left.+\int_{P_{1}}^{\infty} x^{k-\beta}(F(x))^{r-1}(1-F(x))^{n-r} f(x) d x\right\} \\
& +\frac{k}{\nu \beta}\left\{\int_{0}^{Q_{1}} x^{k}(F(x))^{r-1}(1-F(x))^{n-r} f(x) d x\right. \\
& \left.\left.+\int_{P_{1}}^{\infty} x^{k}(F(x))^{r-1}(1-F(x))^{n-r} f(x) d x\right\}-\left(Q_{1}^{k}-P_{1}^{k}\right) p^{r-1} q^{n-r+1}\right],
\end{aligned}
$$

which on simplification and using (3.12) gives

$$
\begin{aligned}
\mu_{r: n}^{(k)}-\mu_{r-1: n-1}^{(k)}= & \frac{k}{n \nu \beta \lambda} \mu_{r: n}^{(k-\beta)}+\frac{k}{n \nu \beta} \mu_{r: n}^{(k)}+\left(\begin{array}{c}
n-1 \\
r-1
\end{array}\right) \\
& \cdot\left[\frac{(Q-p) k}{Q} \int_{0}^{Q_{1}} x^{k-1}(F(x))^{r-1}(1-F(x))^{n-r} d x-\left(Q_{1}^{k}-P_{1}^{k}\right) p^{r-1} q^{n-r+1}\right] .
\end{aligned}
$$

Now, on using the definition of $I_{r: n}^{(k)}$ as given in Lemma 2 and putting the values of $Q_{1}$ and $P_{1}$ in (4.15), we get

$$
\begin{aligned}
\mu_{r: n}^{(k)}-\mu_{r-1: n-1}^{(k)}= & \frac{k}{n \nu \beta \lambda} \mu_{r: n}^{(k-\beta)}+\frac{k}{n \nu \beta} \mu_{r: n}^{(k)}+\frac{(Q-p) k}{Q} I_{r: n}^{(k)} \\
& -\left(\begin{array}{c}
n-1 \\
r-1
\end{array}\right) p^{r-1} q^{n-r+1}\left[\left[\left\{\frac{(1-Q)^{\frac{-1}{\nu}}-1}{\lambda}\right\}^{\frac{1}{\beta}}\right]^{k}-\left[\left\{\frac{(1-P)^{\frac{-1}{\nu}}-1}{\lambda}\right\}^{\frac{1}{\beta}}\right]^{k}\right]
\end{aligned}
$$


where the values of $I_{r: n}^{(k)}$, for different values of $r$, are given in (4.11) and (4.12). This completes the proof of (4.4). Further, on putting $k=\beta$ in (4.4), it reduces to (4.3).

\section{Recursive Algorithm}

Utilizing the knowledge of recurrence relations obtained above one can evaluate the moments of order statistics from mid-truncated Burr XII distribution for $\beta \in Z^{+}, k \geq \beta, \nu>1$ and $\lambda>0$. Algorithm is as following:

Case 1: For $k=m \beta, m \in Z^{+}$

From equation (4.2), we observe that $\mu_{1: n}^{(m \beta)}$ can be evaluated by the knowledge of $\mu_{1: n}^{((m-1) \beta)}$ which in turn can be evaluated with the knowledge of $\mu_{1: n}^{((m-2) \beta)}$ and so on, and after the recursive use of (4.2), $(m-1)$ times, we conclude that $\mu_{1: n}^{(m \beta)}$ comes out to be a function of parameter values only. Thus, if the parameters are known then one can evaluate $\mu_{1: n}^{(k)}$ for all $n$ and $k$ in a simple recursive manner for this case, i.e., for $k=m \beta$.

From equation (4.4), we observe $\mu_{r: n}^{(m \beta)}$ can be evaluated by the knowledge of $\mu_{r-1: n-1}^{(m \beta)}$ and $\mu_{r: n}^{((m-1) \beta)}$ and after $(r-2)$ iterations of equation (4.4) we conclude that to evaluate $\mu_{r: n}^{(m \beta)}$ the required values are $\mu_{1: n-r+1}^{(m \beta)}$, $\mu_{2: n-r+2}^{((m-1) \beta)}, \ldots, \mu_{r: n}^{((m-r+1) \beta)}$.

In particular, to calculate $\mu_{2: n}^{(m \beta)}$ we require the values of $\mu_{1: n-1}^{(m \beta)}$ and $\mu_{2: n}^{((m-1) \beta)}$. As $\mu_{1: n-1}^{(m \beta)}$ is already known and after the recursive use of $(4.2),(m-1)$ times, we conclude that to evaluate $\mu_{2: n}^{(m \beta)}$, the required values are $\mu_{1: n-1}^{(t \beta)}$ for all $t=1,2,3, \ldots, m$, which are already known.

Now for $r=3$ to evaluate $\mu_{3: n}^{(m \beta)}$, for odd $m$, we require the values of $\mu_{1: n-2}^{(m \beta)}, \mu_{1: n-2}^{((m-2) \beta)}, \ldots, \mu_{1: n-2}^{(3 \beta)}$ and $\mu_{2: n-1}^{((m-1) \beta)}, \mu_{2: n-1}^{((m-3) \beta)}, \ldots, \mu_{2: n-1}^{(2 \beta)}$ (which have already been calculated) and $\mu_{3: n}^{(\beta)}$. Using equation (4.3) we can evaluate $\mu_{3: n}^{(\beta)}$, thus by using the values calculated above and utilizing Theorem 2 we can calculate $\mu_{3: n}^{(m \beta)}$ for all odd values of $m$.

Similarly for even values of $m$, one can evaluate $\mu_{3: n}^{(m \beta)}$, by utilizing the above calculated values. In this way one can calculate $\mu_{4: n}^{(m \beta)}, \mu_{5: n}^{(m \beta)}, \ldots$ in a recursive manner.

Case 2: when $k \neq m \beta, m \in Z^{+}$

From equation (4.2) we observe that $\mu_{1: n}^{(k)}$ can be evaluated by the knowledge of $\mu_{1: n}^{(k-\beta)}$ and after the recursive use of (4.2) $(\alpha-1)$ times (where $\alpha$ is the greatest integer satisfying the condition $(k-\alpha \beta)>0$ ), we conclude that $\mu_{1: n}^{(k)}$ is a function of $\mu_{1: n}^{(k-\alpha \beta)}$. Thus in this case, by the knowledge of $\mu_{1: n}^{(k-\alpha \beta)}$ one can evaluate values of $\mu_{1: n}^{(k)}$ for all $n$ and $k$ in a simple recursive manner.

From equation (4.3) we observe that to evaluate $\mu_{r: n}^{(k)}$ we should have the knowledge of only one value with sample size $n$ i.e. $\mu_{r: n}^{(k-\alpha \beta)}$ (where $\alpha$ is the greatest integer satisfying the condition $(k-\alpha \beta)>0$ ) and some moments of lower orders. Using the above information one can calculate $\mu_{2: n}^{(k)}, \mu_{3: n}^{(k)}, \ldots$ in a recursive manner.

\section{Recurrence relations for product moments of order statistics from mid-truncated Burr XII distribution}

\section{Theorem 3}

For $1 \leq r<s \leq n, x<y$ and $j, k>0$, we have

$$
\begin{aligned}
\mu_{r, s: n}^{(j, k)}-\mu_{r, s-1: n}^{(j, k)}= & \frac{k}{(n-s+1) \nu \beta \lambda} \mu_{r, s: n}^{(j, k-\beta)}+\frac{k}{(n-s+1) \nu \beta} \mu_{r, s: n}^{(j, k)} \\
& +C_{r, s: n}^{*}\left[k\left(1-\frac{p}{Q}\right) \int_{0}^{Q_{1}} \int_{x}^{Q_{1}} x^{j} y^{k-1}[F(x)]^{r-1}[F(y)-F(x)]^{s-r-1}\right. \\
& \times[1-F(y)]^{n-s} f(x) d y d x-(1-p)^{n-r} Q_{1}{ }^{k} \sum_{i=0}^{s-r-1}\left(\begin{array}{c}
s-r-1 \\
i
\end{array}\right)(-1)^{i}\left(\frac{p}{q}\right)^{s-r-i-1}
\end{aligned}
$$




$$
\left.\times \int_{0}^{Q_{1}} x^{j}[F(x)]^{r+i-1}[1-F(x)]^{s-r-i-1} f(x) d x\right],
$$

where $C_{r, s: n}^{*}=\frac{n !}{(r-1) !(s-r-1) !(n-s+1) !}$.

Proof

To prove the above theorem, first we evaluate the following term,

$$
\begin{aligned}
E(j, r+i, s) & =\int_{0}^{Q_{1}} x^{j}[F(x)]^{r+i-1}[1-F(x)]^{s-r-i-1} f(x) d x \quad \text { (say) } \\
& =\int_{0}^{Q_{1}} x^{j}[F(x)]^{\alpha-1}[1-F(x)]^{s-\alpha-1} f(x) d x \text {, by taking } r+i=\alpha .
\end{aligned}
$$

From equation (3.5) we know for $x \leq Q_{1}$

$$
\begin{aligned}
& F(x)=\frac{p}{Q}-f(x) \frac{x^{1-\beta}\left(1+\lambda x^{\beta}\right)}{\nu \beta \lambda} \\
\Rightarrow & \frac{p}{Q}-F(x)=f(x) \frac{x^{1-\beta}\left(1+\lambda x^{\beta}\right)}{\nu \beta \lambda} \\
\Rightarrow & \nu \beta \lambda\left[\frac{p}{Q}-F(x)\right] x^{\beta-1}=f(x)\left(1+\lambda x^{\beta}\right) .
\end{aligned}
$$

Now consider,

$$
\begin{aligned}
& E(j, \alpha, s)+\lambda E(j+\beta, \alpha, s)=\int_{0}^{Q_{1}} x^{j}[F(x)]^{\alpha-1}[1-F(x)]^{s-\alpha-1} f(x) d x \\
& +\lambda \int_{0}^{Q_{1}} x^{j+\beta}[F(x)]^{\alpha-1}[1-F(x)]^{s-\alpha-1} f(x) d x \\
& =\int_{0}^{Q_{1}} x^{j}[F(x)]^{\alpha-1}[1-F(x)]^{s-\alpha-1} f(x)\left(1+\lambda x^{\beta}\right) d x \\
& =\nu \beta \lambda \int_{0}^{Q_{1}} x^{j}[F(x)]^{\alpha-1}[1-F(x)]^{s-\alpha-1}\left[\frac{p}{Q}-F(x)\right] x^{\beta-1} d x \text { (using (5.2)) } \\
& =\nu \beta \lambda\left[\frac{p}{Q} \int_{0}^{Q_{1}} x^{j+\beta-1}[F(x)]^{\alpha-1}[1-F(x)]^{s-\alpha-1} d x\right. \\
& \left.-\int_{0}^{Q_{1}} x^{j+\beta-1}[F(x)]^{\alpha}[1-F(x)]^{s-\alpha-1} d x\right] \\
& =\nu \beta \lambda\left[\frac{p}{Q} \frac{I_{\alpha: s-1}^{(j+\beta)}}{\left(\begin{array}{c}
s-2 \\
\alpha-1
\end{array}\right)}-\frac{I_{\alpha+1: s}^{(j+\beta)}}{\left(\begin{array}{c}
s-1 \\
\alpha
\end{array}\right)}\right],
\end{aligned}
$$

where $\alpha=r+i$, and

$$
I_{r: n}^{(k)}=\left(\begin{array}{c}
n-1 \\
r-1
\end{array}\right) \int_{0}^{Q_{1}} x^{k-1}(F(x))^{r-1}(1-F(x))^{n-r} d x,
$$

which is evaluated in Lemma 2.

Proof of the main Theorem 3

Using equation (3.13), and letting $C_{r, s: n}^{*}=\frac{n !}{(r-1) !(s-r-1) !(n-s+1) !}$, consider

$$
\mu_{r, s: n}^{(j, k)}-\mu_{r, s-1: n}^{(j, k)}=\frac{n !}{(r-1) !(s-r-1) !(n-s) !}
$$




$$
\begin{aligned}
\times & {\left[\int_{0}^{Q_{1}} \int_{x}^{Q_{1}} x^{j} y^{k}[F(x)]^{r-1}[F(y)-F(x)]^{s-r-1}[1-F(y)]^{n-s} f(x) f(y) d y d x\right.} \\
& \left.+\int_{P_{1}}^{\infty} \int_{x}^{\infty} x^{j} y^{k}[F(x)]^{r-1}[F(y)-F(x)]^{s-r-1}[1-F(y)]^{n-s} f(x) f(y) d y d x\right] \\
- & \frac{n !}{(r-1) !(s-r-2) !(n-s+1) !} \\
\times & {\left[\int_{0}^{Q_{1}} \int_{x}^{Q_{1}} x^{j} y^{k}[F(x)]^{r-1}[F(y)-F(x)]^{s-r-2}[1-F(y)]^{n-s+1} f(x) f(y) d y d x\right.} \\
& \left.+\int_{P_{1}}^{\infty} \int_{x}^{\infty} x^{j} y^{k}[F(x)]^{r-1}[F(y)-F(x)]^{s-r-2}[1-F(y)]^{n-s+1} f(x) f(y) d y d x\right] \\
= & C_{r, s: n}^{*}\left[\int_{0}^{Q_{1}} \int_{x}^{Q_{1}} x^{j} y^{k}[F(x)]^{r-1}[F(y)-F(x)]^{s-r-2}[1-F(y)]^{n-s} f(x) f(y)\right. \\
\times & \{(n-s+1)(F(y)-F(x))-(s-r-1)(1-F(y))\} d y d x \\
+ & \int_{P_{1}}^{\infty} \int_{x}^{\infty} x^{j} y^{k}[F(x)]^{r-1}[F(y)-F(x)]^{s-r-2}[1-F(y)]^{n-s} f(x) f(y) \\
\times & \{(n-s+1)(F(y)-F(x))-(s-r-1)(1-F(y))\} d y d x] \\
= & C_{r, s: n}^{*}\left[\int_{0}^{Q_{1}} \int_{x}^{Q_{1}} x^{j} y^{k}[F(x)]^{r-1}[F(y)-F(x)]^{s-r-2}[1-F(y)]^{n-s} f(x) f(y)\right. \\
\times & \times\{(n-r) F(y)-(n-s+1) F(x)-(s-r-1)\} d y d x \\
\times & \int_{P_{1}}^{\infty} \int_{x}^{\infty} x^{j} y^{k}[F(x)]^{r-1}[F(y)-F(x)]^{s-r-2}[1-F(y)]^{n-s} f(x) f(y) \\
\times & (n-r) F(y)-(n-s+1) F(x)-(s-r-1)\} d y d x] .
\end{aligned}
$$

Let

$$
\varphi(x, y)=-[F(y)-F(x)]^{s-r-1}[1-F(y)]^{n-s+1} .
$$

Then,

$$
\begin{aligned}
\frac{\partial \varphi(x, y)}{\partial y}= & -(s-r-1)[F(y)-F(x)]^{s-r-2}[1-F(y)]^{n-s+1} f(y) \\
& +(n-s+1)[F(y)-F(x)]^{s-r-1}[1-F(y)]^{n-s} f(y) \\
= & {[F(y)-F(x)]^{s-r-2}[1-F(y)]^{n-s} f(y)[(n-r) F(y)} \\
& -(n-s+1) F(x)-(s-r-1)] .
\end{aligned}
$$

Putting the above value in (5.3), we get

$$
\begin{aligned}
\mu_{r, s: n}^{(j, k)}-\mu_{r, s-1: n}^{(j, k)}= & C_{r, s: n}^{*}\left[\int_{0}^{Q_{1}} \int_{x}^{Q_{1}} x^{j} y^{k}[F(x)]^{r-1} \frac{\partial \varphi(x, y)}{\partial y} f(x) d y d x\right. \\
& \left.+\int_{P_{1}}^{\infty} \int_{x}^{\infty} x^{j} y^{k}[F(x)]^{r-1} \frac{\partial \varphi(x, y)}{\partial y} f(x) d y d x\right] \\
& =C_{r, s: n}^{*}\left[\int_{0}^{Q_{1}} x^{j}[F(x)]^{r-1}\left(\int_{x}^{Q_{1}} y^{k} \frac{\partial \varphi(x, y)}{\partial y} d y\right) f(x) d x\right.
\end{aligned}
$$




$$
\left.+\int_{P_{1}}^{\infty} x^{j}[F(x)]^{r-1}\left(\int_{x}^{\infty} y^{k} \frac{\partial \varphi(x, y)}{\partial y} d y\right) f(x) d x\right]
$$

Now, consider

$$
\int_{x}^{Q_{1}} y^{k} \frac{\partial \varphi(x, y)}{\partial y} d y=Q_{1}^{k} \varphi\left(x, Q_{1}\right)+k \int_{x}^{Q_{1}} y^{k-1}[F(y)-F(x)]^{s-r-1}[1-F(y)]^{n-s+1} d y .
$$

From equations (5.4) and (3.9), we have

$$
\varphi\left(x, Q_{1}\right)=-[p-F(x)]^{s-r-1}[1-p]^{n-s+1} .
$$

Putting (5.8) in equation (5.7), we get

$$
\begin{aligned}
\int_{x}^{Q_{1}} y^{k} \frac{\partial \varphi(x, y)}{\partial y} d y= & k \int_{x}^{Q_{1}} y^{k-1}[F(y)-F(x)]^{s-r-1}[1-F(y)]^{n-s+1} d y \\
& -Q_{1}{ }^{k}[p-F(x)]^{s-r-1}[1-p]^{n-s+1}
\end{aligned}
$$

also,

$$
\int_{x}^{\infty} y^{k} \frac{\partial \varphi(x, y)}{\partial y} d y=k \int_{x}^{\infty} y^{k-1}[F(y)-F(x)]^{s-r-1}[1-F(y)]^{n-s+1} d y .
$$

Putting (5.9) and (5.10) in equation (5.6), we get

$$
\begin{aligned}
\mu_{r, s: n}^{(j, k)}-\mu_{r, s-1: n}^{(j, k)}= & C_{r, s: n}^{*}\left[\int _ { 0 } ^ { Q _ { 1 } } x ^ { j } [ F ( x ) ] ^ { r - 1 } \left\{k \int_{x}^{Q_{1}} y^{k-1}[F(y)-F(x)]^{s-r-1}[1-F(y)]^{n-s+1} d y\right.\right. \\
& \left.-Q_{1}{ }^{k}[p-F(x)]^{s-r-1}[1-p]^{n-s+1}\right\} f(x) d x \\
& \left.+\int_{P_{1}}^{\infty} x^{j}[F(x)]^{r-1}\left\{k \int_{x}^{\infty} y^{k-1}[F(y)-F(x)]^{s-r-1}[1-F(y)]^{n-s+1} d y\right\} f(x) d x\right] \\
= & C_{r, s: n}^{*}\left[k \int_{0}^{Q_{1}} \int_{x}^{Q_{1}} x^{j} y^{k-1}[F(x)]^{r-1}[F(y)-F(x)]^{s-r-1}[1-F(y)]^{n-s+1} f(x) d y d x\right. \\
& +k \int_{P_{1}}^{\infty} \int_{x}^{\infty} x^{j} y^{k-1}[F(x)]^{r-1}[F(y)-F(x)]^{s-r-1}[1-F(y)]^{n-s+1} f(x) d y d x \\
& \left.-\int_{0}^{Q_{1}} x^{j} Q_{1}{ }^{k}[p-F(x)]^{s-r-1}[1-p]^{n-s+1}[F(x)]^{r-1} f(x) d x\right] .
\end{aligned}
$$

Considering the last term of the above equation and writing $(n-s+1)=(n-r)-(s-r-1)$, we get

$$
\begin{aligned}
Q_{1}{ }^{k} & {[1-p]^{n-s+1} \int_{0}^{Q_{1}} x^{j}[p-F(x)]^{s-r-1}[F(x)]^{r-1} f(x) d x } \\
& =Q_{1}{ }^{k}[1-p]^{n-r} \int_{0}^{Q_{1}} x^{j} \frac{[p-F(x)]^{s-r-1}}{(1-p)^{s-r-1}}[F(x)]^{r-1} f(x) d x \\
& =Q_{1}{ }^{k}[1-p]^{n-r} \int_{0}^{Q_{1}} x^{j}\left(\frac{p-F(x)}{q}\right)^{s-r-1}[F(x)]^{r-1} f(x) d x \\
& =Q_{1}{ }^{k}[1-p]^{n-r} \int_{0}^{Q_{1}} x^{j}[F(x)]^{r-1}\left(\frac{p(1-F(x))}{q}-F(x)\right)^{s-r-1} f(x) d x .
\end{aligned}
$$


Expanding $\left(\frac{p}{q}(1-F(x))-F(x)\right)^{s-r-1}$ binomially we get the above expression equal to

$$
\begin{aligned}
& Q_{1}{ }^{k} {[1-p]^{n-r} \int_{0}^{Q_{1}} x^{j}[F(x)]^{r-1} \sum_{i=0}^{s-r-1}\left(\begin{array}{c}
s-r-1 \\
i
\end{array}\right)(-F(x))^{i}\left(\frac{p}{q}(1-F(x))\right)^{s-r-i-1} f(x) d x } \\
&=Q_{1}{ }^{k}[1-p]^{n-r} \sum_{i=0}^{s-r-1}\left(\begin{array}{c}
s-r-1 \\
i
\end{array}\right)\left(\frac{p}{q}\right)^{s-r-i-1}(-1)^{i} \int_{0}^{Q_{1}} x^{j}[F(x)]^{r+i-1}[1-F(x)]^{s-r-i-1} f(x) d x .
\end{aligned}
$$

Putting (5.12) in (5.11), we get

$$
\begin{aligned}
& \mu_{r, s: n}^{(j, k)}-\mu_{r, s-1: n}^{(j, k)} \\
& =C_{r, s: n}^{*}\left[k \int_{0}^{Q_{1}} \int_{x}^{Q_{1}} x^{j} y^{k-1}[F(x)]^{r-1}[F(y)-F(x)]^{s-r-1}[1-F(y)]^{n-s+1} f(x) d y d x\right. \\
& \quad+k \int_{P_{1}}^{\infty} \int_{x}^{\infty} x^{j} y^{k-1}[F(x)]^{r-1}[F(y)-F(x)]^{s-r-1}[1-F(y)]^{n-s+1} f(x) d y d x \\
& \left.\quad-Q_{1}{ }^{k}[1-p]^{n-r} \sum_{i=0}^{s-r-1}\left(\begin{array}{c}
s-r-1 \\
i
\end{array}\right)\left(\frac{p}{q}\right)^{s-r-i-1}(-1)^{i} \int_{0}^{Q_{1}} x^{j}[F(x)]^{r+i-1}[1-F(x)]^{s-r-i-1} f(x) d x\right] .
\end{aligned}
$$

Using (3.5) in (5.13), we get

$$
\begin{aligned}
& \mu_{r, s: n}^{(j, k)}-\mu_{r, s-1: n}^{(j, k)}=C_{r, s: n}^{*}\left[k\left(1-\frac{p}{Q}\right) \int_{0}^{Q_{1}} \int_{x}^{Q_{1}} x^{j} y^{k-1}[F(x)]^{r-1}[F(y)-F(x)]^{s-r-1}[1-F(y)]^{n-s} f(x) d y d x\right. \\
& +\frac{k}{\nu \beta \lambda} \int_{0}^{Q_{1}} \int_{x}^{Q_{1}} x^{j} y^{k-\beta}[F(x)]^{r-1}[F(y)-F(x)]^{s-r-1}[1-F(y)]^{n-s} f(x) f(y) d y d x \\
& +\frac{k}{\nu \beta} \int_{0}^{Q_{1}} \int_{x}^{Q_{1}} x^{j} y^{k}[F(x)]^{r-1}[F(y)-F(x)]^{s-r-1}[1-F(y)]^{n-s} f(x) f(y) d y d x \\
& +\frac{k}{\nu \beta \lambda} \int_{P_{1}}^{\infty} \int_{x}^{\infty} x^{j} y^{k-\beta}[F(x)]^{r-1}[F(y)-F(x)]^{s-r-1}[1-F(y)]^{n-s} f(x) f(y) d y d x \\
& +\frac{k}{\nu \beta} \int_{P_{1}}^{\infty} \int_{x}^{\infty} x^{j} y^{k}[F(x)]^{r-1}[F(y)-F(x)]^{s-r-1}[1-F(y)]^{n-s} f(x) f(y) d y d x \\
& -(1-p)^{n-r} Q_{1}{ }^{k} \sum_{i=0}^{s-r-1}\left(\begin{array}{c}
s-r-1 \\
i
\end{array}\right)(-1)^{i}\left(\frac{p}{q}\right)^{s-r-i-1} \int_{0}^{Q_{1}} x^{j}[F(x)]^{r+i-1} \\
& \left.\times[1-F(x)]^{s-r-i-1} f(x) d x\right] \\
& =\frac{k}{(n-s+1) \nu \beta \lambda} \mu_{r, s: n}^{(j, k-\beta)}+\frac{k}{(n-s+1) \nu \beta} \mu_{r, s: n}^{(j, k)} \\
& +C_{r, s: n}^{*}\left[k\left(1-\frac{p}{Q}\right) \int_{0}^{Q_{1}} \int_{x}^{Q_{1}} x^{j} y^{k-1}[F(x)]^{r-1}[F(y)-F(x)]^{s-r-1}\right. \\
& \times[1-F(y)]^{n-s} f(x) d y d x-(1-p)^{n-r} Q_{1} \sum_{i=0}^{s-r-1}\left(\begin{array}{c}
s-r-1 \\
i
\end{array}\right)(-1)^{i}\left(\frac{p}{q}\right)^{s-r-i-1} \\
& \left.\times \int_{0}^{Q_{1}} x^{j}[F(x)]^{r+i-1}[1-F(x)]^{s-r-i-1} f(x) d x\right],
\end{aligned}
$$

Stat., Optim. Inf. Comput. Vol. 7, March 2019 
which completes the proof of Theorem 3 .

Remark 1

Proceeding in a similar manner as in Section 3, and considering the following baseline distributions, which are particular cases of Burr XII distribution, namely

(i) Lomax distribution:

$$
F(x)=1-\left[1+\frac{x}{a}\right]^{-\nu}
$$

(ii) Weibull distribution:

$$
F(x)=1-e^{-x^{\beta}}
$$

(iii) Compound Weibull or Weibull-Gamma distribution:

$$
F(x)=1-\left[1+\frac{x^{\beta}}{\delta}\right]^{-\nu}
$$

(iv) Weibull-Exponential distribution:

$$
F(x)=\frac{x^{\beta} / \delta}{\left(1+x^{\beta} / \delta\right)}
$$

(v) Log logistic distribution:

$$
F(x)=\frac{(x / a)^{\beta}}{1+(x / a)^{\beta}}
$$

(vi) Exponential distribution:

$$
F(x)=1-e^{-x}
$$

(vii) Rayleigh distribution:

$$
F(x)=1-e^{-x^{2} / 2}
$$

(viii) Generalized Rayleigh distribution:

$$
F(x)=1-\left[1+\alpha x^{2} / 2\right]^{-(1 / \alpha)}
$$

(ix) Generalized Pareto distribution:

$$
F(x)=1-[1+p x]^{-(1 / p)},
$$

we can construct the corresponding mid-truncated distributions and find corresponding recurrence relations for moments of order statistics arising from these distributions.

Thus, the recurrence relations obtained in Sections 4 and 5 are also true for mid-truncated

Lomax $(\beta=1, \lambda=1 / a)$, Weibull $(\nu=1 / \lambda, \lambda \rightarrow 0)$, Weibull-Gamma $(\lambda=1 / \delta)$, Weibull-Exponential $(\nu=$ $1, \lambda=1 / \delta)$, Log logistic $\left(\nu=1, \lambda=a^{-\beta}\right)$, Exponential $(\nu=1 / \lambda, \beta=1, \lambda \rightarrow 0)$, Rayleigh $(\beta=2, \nu=1 /(2 \lambda)$, $\lambda \rightarrow 0)$, Generalized Rayleigh $(\beta=2, \nu=1 / \alpha, \lambda=\alpha / 2)$ and Generalized Pareto $(\beta=1, \nu=1 / p, \lambda=p)$ distributions.

\section{Remark 2}

The results deduced for the mid-truncated Weibull distribution, so obtained, are in agreement with the results of Okasha et al. [14]. 


\section{Characterization of mid-truncated distributions using order statistics}

In this section we will use the conditional moments of order statistics to characterize mid-truncated distributions.

Let $X_{1}, X_{2}, X_{3}, \ldots, X_{n}$ be a random samples of size $n$ from the distribution defined in (3.3) and $X_{1: n} \leq$ $X_{2: n} \leq \ldots \leq X_{n: n}$ be the corresponding order statistics. Then the probability density function (pdf) of $X_{r: n}$ $(1 \leq r \leq n)$ is given by $f_{r: n}(x)$ as defined by equation (3.10) and joint probability density function of $X_{r: n}$ and $X_{s: n}(1 \leq r<s \leq n)$ is given by $f_{r, s: n}(x, y)$ as defined in (3.11).

Many authors have studied characterizations depending on order statistics. Among those Khan and Khan [8] characterized Burr XII distribution through moments of order statistics. Lin [9] used product moments to characterize power function, exponential and normal distributions. For extensive survey refer to Huang and Lin [7] and Huang [6].

Next, we establish a theorem to characterize mid-truncated distributions based on order statistics.

\section{Theorem 4}

Let $X$ be a random variable having probability density function as (2.1), then $X$ is having mid-truncated distribution iff

$$
E\left(X_{r+1: n}^{k} \mid X_{r: n}=x\right)=x^{k}+\frac{k \Delta(x)}{(1-F(x))^{n-r}},
$$

where

$$
\Delta(x)=\int_{x}^{\infty} y^{k-1}\left[1-\left[\frac{p}{G\left(Q_{1}\right)} \int_{-\infty}^{y} g(t) d t+\frac{p}{G\left(Q_{1}\right)} \int_{-\infty}^{Q_{1}} g(t) d t+\frac{q}{\left(1-G\left(P_{1}\right)\right)} \int_{P_{1}}^{y} g(t) d t\right]\right]^{n-r} d y,
$$

and $F(x)$ is the cumulative distribution function of the mid-truncated random variable.

Proof

Necessary condition:

We have

$$
\begin{aligned}
E\left(X_{r+1: n}^{k} \mid X_{r: n}=x\right) & =\frac{C_{r, r+1: n}}{C_{r: n}}\left[\int_{x}^{\infty} \frac{y^{k}(F(x))^{r-1}(1-F(y))^{n-r-1} f(x) f(y)}{(F(x))^{r-1}(1-F(x))^{n-r} f(x)} d y\right] \\
& =\frac{(n-r)}{(1-F(x))^{n-r}} \int_{x}^{\infty} y^{k}(1-F(y))^{n-r-1} f(y) d y,
\end{aligned}
$$

where $X_{r+1: n}=y$. Integrating by parts, we get

$$
\begin{aligned}
E\left(X_{r+1: n}^{k} \mid X_{r: n}=x\right)= & \frac{(n-r)}{(1-F(x))^{n-r}}\left(\left.\frac{-y^{k}(1-F(y))^{n-r}}{n-r}\right|_{x} ^{\infty}+k \int_{x}^{\infty} \frac{y^{k-1}(1-F(y))^{n-r}}{n-r} d y\right) \\
= & x^{k}+\frac{k}{(1-F(x))^{n-r}} \int_{x}^{\infty} y^{k-1}\left[1-\left[\frac{p}{G\left(Q_{1}\right)} \int_{-\infty}^{y} g(t) d t+\frac{p}{G\left(Q_{1}\right)} \int_{-\infty}^{Q_{1}} g(t) d t\right.\right. \\
& \left.\left.+\frac{q}{\left(1-G\left(P_{1}\right)\right)} \int_{P_{1}}^{y} g(t) d t\right]\right]^{n-r} d y \\
= & x^{k}+\frac{k \Delta(x)}{(1-F(x))^{n-r}} .
\end{aligned}
$$

Hence necessary part is proved.

Sufficient condition:

Let

$$
E\left(X_{r+1: n}^{k} \mid X_{r: n}=x\right)=x^{k}+\frac{k \Delta(x)}{(1-F(x))^{n-r}},
$$


then

$$
\frac{(n-r)}{(1-F(x))^{n-r}} \int_{x}^{\infty} y^{k}(1-F(y))^{n-r-1} f(y) d y=x^{k}+\frac{k \Delta(x)}{(1-F(x))^{n-r}} .
$$

Multiplying both sides by $(1-F(x))^{n-r}$, and differentiating both sides with respect to $x$, we get

$$
\begin{aligned}
& -(n-r) x^{k}(1-F(x))^{n-r-1} f(x) \\
& =k x^{k-1}(1-F(x))^{n-r}-(n-r) x^{k}(1-F(x))^{n-r-1} f(x) \\
& \quad-k x^{k-1}\left[1-\left[\frac{p}{G\left(Q_{1}\right)} \int_{-\infty}^{y} g(t) d t+\frac{p}{G\left(Q_{1}\right)} \int_{-\infty}^{Q_{1}} g(t) d t+\frac{q}{\left(1-G\left(P_{1}\right)\right)} \int_{P_{1}}^{y} g(t) d t\right]\right]^{n-r} .
\end{aligned}
$$

On simplification, we get

$$
F(x)=\frac{p}{G\left(Q_{1}\right)} \int_{-\infty}^{y} g(t) d t+\frac{p}{G\left(Q_{1}\right)} \int_{-\infty}^{Q_{1}} g(t) d t+\frac{q}{\left(1-G\left(P_{1}\right)\right)} \int_{P_{1}}^{y} g(t) d t .
$$

This is the cumulative distribution function of the mid-truncated random variable.

Hence sufficient part is proved.

By applying the above theorem, $X$ has mid-truncated Burr XII distribution iff

$$
E\left(X_{r+1: n}^{k} \mid X_{r: n}=x\right)=x^{k}+\frac{k \Delta(x)}{(1-F(x))^{n-r}},
$$

where

$$
\begin{aligned}
& F(x)=p \frac{1-\left(1+\lambda x^{\beta}\right)^{-\nu}}{1-\left(1+\lambda Q_{1}^{\beta}\right)^{-\nu}} I_{0<x \leq Q_{1}}+\left(1-q \frac{\left(1+\lambda x^{\beta}\right)^{-\nu}}{\left(1+\lambda P_{1}^{\beta}\right)^{-\nu}}\right) I_{P_{1} \leq x<\infty} \\
& \Delta(x)=\int_{x}^{\infty} y^{k-1}\left[1-\left[p\left\{1+\frac{1-\left(1+\lambda x^{\beta}\right)^{-\nu}}{1-\left(1+\lambda Q_{1}^{\beta}\right)^{-\nu}}\right\}+q\left\{\frac{\left(1+\lambda P_{1}^{\beta}\right)^{-\nu}-\left(1+\lambda y^{\beta}\right)^{-\nu}}{\left(1+\lambda P_{1}^{\beta}\right)^{-\nu}}\right\}\right]\right]^{n-r} d y \\
& I_{0<x \leq Q_{1}}= \begin{cases}1, & 0<x \leq Q_{1} \\
0, & \text { elsewhere }\end{cases}
\end{aligned}
$$

and

$$
I_{P_{1} \leq x<\infty}= \begin{cases}1, & P_{1} \leq x<\infty \\ 0, & \text { elsewhere }\end{cases}
$$

\section{Conclusion}

In live models as discussed earlier in the paper, mid-truncated Burr distribution is quite effectively used when the random variable is restricted to be observed on some sub intervals of the given specified range. Figures 1 and 2 depict the probability density function of Mid-truncated Burr distribution for different values of parameters and for different points of truncation.

We have derived the recurrence relations for single moments and product moments of order statistics from midtruncated Burr XII distribution, and utilizing the knowledge of recurrence relations we can evaluate the moments of order statistics using the recursive algorithm as discussed in Section 4. Using these moments, to investigate the effect of the shape parameters $\beta, \nu$ and scale parameter $\lambda$ on the mid-truncated Burr XII density function, we have computed mean, variance, skewness and kurtosis for different values of the parameters, which are presented in 
Tables 1 to 3. From these tables we observe that by keeping any two parameters fixed, the increase in the value of the third parameter results in the decrease of the values of the measures calculated.

Also, due to the flexibility of the considered distribution, i.e., mid-truncated Burr XII distribution, which contains, as special sub models, mid-truncated Weibull distribution, mid-truncated exponential distribution, midtruncated log-logistic distribution, among others, in accommodating different forms of risk functions, this midtruncated Burr XII distribution is appropriate for a variety of problems modelling lifetime data.

\section{Acknowledgement}

Authors are grateful to the Coordinating Editor and the learned referee for giving valuable comments which led to an improvement in the presentation of the paper.

\section{REFERENCES}

1. N. Balakrishnan, and P.C. Joshi, Moments of order statistics from doubly truncated power function distribution, Aligarh Journal of Statistics, vol. 1, no. 2, pp. 1498-1505, 1981.

2. N. Balakrishnan, H.J. Malik, and S. Ahmed, Recurrence relations and identities for moment of order statistics-II: Specific continuous distributions, Commun. Statist. Theory Meth., vol. 17, no. 8, pp. 2657-2694, 1988.

3. I.W. Burr, Cumulative frequency functions, Annals of Mathematical Statistics, vol. 13, pp. 215-232, 1942.

4. H.A. David and H.N. Nagaraja, Order Statistics, Third Edition, John Wiley \& Sons, 2003.

5. M. Hashempour, Evidences in lifetimes of sequential r-out-of $n$ systems and optimal sample size determination for Burr XII populations, Statistics Opt. Inform. Comput., vol. 5, pp. 147-157, 2017.

6. J.S. Huang, Moment problem of order statistics: A review, Inter. Statist. Rev., vol. 57, pp. 59-66, 1989.

7. J.S. Huang, and G.D. Lin, Characterizations of distributions by linear combinations of order statistics, Bull. Inst. Math., Academia Sinica, vol. 12, pp. 179-202, 1984.

8. A.H. Khan, and I.A. Khan, Moments of order statistics from Burr XII distribution and its characterizations, Metron, vol. XLV-N1-2, no. 30, pp. 21-29, 1987.

9. G.D. Lin, The product moments of order statistics with applications to characterizations of distributions, J. Statist. Plan. Infer., vol. 21, pp. 395-406, 1989.

10. H.J. Malik, Exact moments of order statistics from a power function distribution, Secand. Act. J., pp. 64-69, 1967.

11. M.M. Mohie El-Din, M.A.W. Mahmoud, and K.S. Sultan, On moment generating function of order statistics for doubly truncated exponential distribution, Metron, vol. LIII, pp. 171-183, 1995.

12. M.M. Mohie El-Din, and K.S. Sultan, Recurrence relations between any continuous function of order statistics for doubly truncated continuous distributions and its applications, The Egyptian Statistical Journal, vol. 37, pp. 336-345, 1993.

13. M.M. Mohie El-Din, M.M. Amein, and A.M. Abd El-Raheem, On mid-truncated distributions and its applications, J. Adv. Res. App. Math., vol. 5, no. 2, pp. 20-38, 2013.

14. H. Okasha, M.M. Mohie-El-Din, and M. Mahmoud, On mid-truncated distributions and its applications in order statistic, J. Adv. Res. Stat. Probab., vol. 3, no. 2, pp. 47-57, 2011.

15. A. Pathak, and A. Chaturvedi, Estimation of the reliability function for two-parameter exponentiated Rayleigh or Burr type $X$ distribution, Statistics Opt. Inform. Comput., vol. 2, pp. 305-322, 2014.

16. K. Pearson, E.S. Pearson, and N.L. Johnson, Tables of the incomplete beta function, Second Edition, 1968.

17. J. Saran, and N. Pushkarna, Moments of order statistics from doubly truncated generalized exponential distribution, Aligarh Journal of Statistics, vol. 19, pp. 77-82, 1999.

18. J. Saran, and N. Pushkarna, Moments of order statistics from doubly truncated linear-exponential distribution, Journal of the Korean Statistical Society, vol. 28, no. 3, pp. 279-296, 1999.

19. J. Saran, and N. Pushkarna, Recurrence relations for moments of progressive type-II right censored order statistics from Burr distribution, Statistics: Journal of theoretical and Applied Statistics, vol. 35, no. 4, pp. 495-507, 2001.

20. A.A. Soliman, Estimation of parameters of life from progressively censored data using Burr-XII Model, IEEE Transactions on Reliability, vol. 54, pp. 34-42, 2005.

21. G.J. Tortora, B.R. Funke, and C.L. Case, Microbiology An Introduction, Eighth Edition, Pearson Education, 2004. 